15

\title{
Болометрические приемники инфракрасного и терагерцового излучения с поглотителями, обладающими частотной дисперсией проводимости
}

\author{
(C) М.А. Демьяненко
}

Институт фозики полупроводников им. А.В. Ржанова СО РАН, 630090 Новосибирск, Россия

e-mail: demyanenko@isp.nsc.ru

(Поступило в Редакцию 12 февраля 2018 г.)

Применение тонких металлических поглотителей позволяет существенно повысить коэффициент поглощения и чувствительность матричных микроболометрических приемников в терагерцовом диапазоне. Показано, что поглотители, обладающие частотной дисперсией проводимости, в отличие от традиционно применяемых бездисперсионных поглотителей, позволяют достичь практически полного поглощения инфракрасного излучения при сохранении повышенной чувствительности в терагерцовом диапазоне.

\section{Введение}

Неохлаждаемые болометрические приемники активно применяются для регистрации и визуализации излучения терагерцового $(\mathrm{THz})$ спектрального диапазона. Первые системы визуализации с активной подсветкой на длине волны $\sim 100 \mu \mathrm{m}$ были реализованы с использованием газовых [1] и квантово-каскадных [2] лазеров, а также лазеров на свободных электронах [3]. Применяемые в этих работах микроболометры были разработаны для инфракрасного (ИК) диапазона $8-14 \mu \mathrm{m}$, в котором они обладают высоким коэффициентом поглощения излучения, практически равным единице, и высокой чувствительностью - мощность, эквивалентная шуму (Noise Equivalent Power, NEP), падающая на один пиксель, может составлять около $10 \mathrm{pW}$ [4]. Однако на длинах волн, близких к $100 \mu \mathrm{m}$, их чувствительность оказалась много ниже - NEP $=200-300 \mathrm{pW}$ [4], что, в частности, обусловлено малым коэффициентом поглощением терагерцового излучения в микроболометpax $(\sim 3-4 \%)$. В дальнейшем величина коэффициента поглощения была значительно повышена путем замены германиевого окна на кремниевое и использования дополнительного тонкого металлического поглотителя, нанесенного на термочувствительную мембрану микроболометра, что позволило понизить NEP до 40 pW [4]. Однако применение металлического поглотителя примерно вдвое понизило чувствительность болометров в полосе 8-14 $\mu \mathrm{m} \mathrm{[5].}$

Увеличение эффективной толщины оптического резонатора, расположенного между поглотителем и отражателем, например, с помощью нанесенного на отражатель слоя нитрида кремния толщиной $9 \mu \mathrm{m}$ [6], или применение дополнительного оптического резонатора, образованного $50 \mu \mathrm{m}$ вакуумным зазором между микроболометром и входным кремниевым окном [7], привело к дальнейшему понижению NEP вплоть до $\sim 17 \mathrm{pW}$ на длине волны $97 \mu \mathrm{m}$ [7]. Однако при этом в обоих случаях приемник становится более узкополосным. В работе [8] предложен инвертированный тип конструкции болометрического приемника терагерцового излучения, в котором в отличие от традиционного типа излучение падает не со стороны прилегающего к болометру вакуума, а со стороны прозрачной подложки, на которой он изготовлен. Применение дополнительного оптического резонатора в инвертированных болометрах позволяет получать коэффициенты поглощения, близкие к 100\%, при этом такие болометры остаются широкополосными. В частности, инвертированный тип болометра может обеспечить высокоэффективное поглощение излучения (более 80\%) в диапазоне длин волн от 37 до $200 \mu \mathrm{m}$. Однако для изготовления матричных приемников такого типа требуется изменение существующей технологии кремниевых мультиплексоров.

В настоящей работе проводится анализ влияния частотной дисперсии проводимости поглотителя на спектральную зависимость коэффициента поглощения инфракрасного и терагерцового излучения в болометрических приемниках традиционного типа. Показана возможность повышения коэффициента поглощения в ИК области (вблизи $10 \mu \mathrm{m}$ ) практически до $100 \%$ при сохранении повышенной чувствительности в $\mathrm{THz}$ области (вблизи $100 \mu \mathrm{m}$ ) без применения дополнительных резонаторов и при использовании высоты подвески болометpa, не превышающей 4-5 $\mu \mathrm{m}$. Последнее обстоятельство обеспечивает высокий процент выхода годных приемников, так как резкое возрастание количества дефектных микроболометров происходит при увеличении высоты подвески до $8 \mu \mathrm{m}$ и выше [6].

\section{Основные соотношения для расчета коэффициента поглощения в болометрическом приемнике с поглотителем, обладающим частотной дисперсией проводимости}

Расчет спектральных зависимостей коэффициента поглощения инфракрасного и терагерцового излучения бу- 
дем проводить матричным методом [9], детали которого применительно к болометрическим структурам с поглотителем и отражателем обладающими произвольной толщиной и комплексной проводимостью, изложены в работе [10]. Здесь с целью проведения качественного анализа влияния частотной дисперсии проводимости поглотителя на спектральную зависимость коэффициента поглощения приведем упрощенное соотношение, применимое к болометру традиционного типа в приближении малой толщины мембраны и идеального отражателя. В таком болометре коэффициент поглощения TE-волны, распространяющейся под углом $\theta_{v}$ к нормали поверхности болометра, задается соотношением

$$
A=\frac{4 p_{v} f_{a 1}}{\left(Y_{g 2}+f_{a 2}\right)^{2}+\left(p_{v}+f_{a 1}\right)^{2}} .
$$

Здесь $p_{v}=\left(\varepsilon_{0} / \mu_{0}\right)^{1 / 2} \cos \theta_{v}-$ адмиттанс распространяющейся в вакууме ТЕ-волны, $\varepsilon_{0}, \mu_{0}$ - абсолютные диэлектрическая и магнитная проницаемости вакуума, $f_{a 1}, f_{a 2}-$ активная и реактивная компоненты слоевой проводимости поглотителя на частоте излучения, $Y_{g 2}-$ реактивная компонента адмиттанса оптического резонатора $Y_{g}$, состоящего из вакуумного зазора толщиной $d_{g}$, расположенного между отражателем и мембраной с поглотителем. В данном приближении адмиттанс $Y_{g}$ является чисто реактивным $Y_{g}=i p_{v} \operatorname{ctg}\left(\kappa_{v} d_{g} \cos \theta_{v}\right)$, где $i-$ мнимая единица, $\kappa_{v}=(2 \pi / \lambda)\left(\varepsilon_{0} \mu_{0}\right)^{1 / 2}, \lambda-$ длина волны излучения в вакууме. Отметим, что соотношение (1) может быть получено непосредственно из известного соотношения для амплитудного коэффициента отражения $r=\left(p_{v}-Y_{b}\right) /\left(p_{v}+Y_{b}\right)$, если учесть, что адмиттанс болометра $Y_{b}$ в приближении тонкого поглотителя может быть записан в виде $Y_{b}=Y_{g}+f_{a 1}+i f_{a 2}$ [10] и что при идеальном отражателе $A=1-|r|^{2}$.

В общем случае при наличии частотной дисперсии реактивная компонента слоевой проводимости поглотителя $f_{a 2}$ не равна нулю и, как следует из соотношения (1), полное поглощение излучения происходит при выполнении двух условий

$$
Y_{g 2}=-f_{a 2} \text { и } f_{a 1}=p_{v} .
$$

При отсутствии частотной дисперсии проводимости поглотителя $f_{a 2}=0$ и полное поглощение падающего на болометр излучения происходит при выполнении известных условий, которые при $\theta_{v}=0$ выполняются для длины волны $\lambda=4 d_{g} /(1+2 m)$ и слоевого сопротивления поглотителя $R_{a}=1 / f_{a 1}=$ $=1 / Y_{v}=Z_{v}=\left(\mu_{0} / \varepsilon_{0}\right)^{1 / 2}=377 \Omega / \square$, где $m$ - натуральное число, $Y_{v}$ и $Z_{v}$ - адмиттанс и импеданс вакуума соответственно. Как и в работе [10], для ТМ-волны во всех приведенных выше соотношениях необходимо заменить $p_{v}$ на $p_{v}^{\prime}=\left(\varepsilon_{0} / \mu_{0}\right)^{1 / 2} / \cos \theta_{v}$.

Во введении было отмечено, что применение металлического поглотителя излучения для повышения коэффициента поглощения в $\mathrm{THz}$ области приводит к понижению чувствительности болометра в ИК области.
Это обусловлено тем, что поглотитель обычно изготавливается из высокоомных металлов или их сплавов, которые характеризуются малым временем релаксации электронов и соответственно их электропроводность практически не зависит от частоты в ИК и $\mathrm{THz}$ диапазоне. Действительно, в терагерцовом диапазоне при высоте подвески болометра $d_{g}$, не превышающей 4-5 $\mu \mathrm{m}$, выполняется условие $\lambda \gg d_{g}$ и нормированная реактивная компонента адмиттанса оптического резонатора $Y_{g 2} / p_{v}$ становится значительно больше единицы. Для достижения максимального коэффициента поглощения в $\mathrm{THz}$ диапазоне величина слоевой проводимости поглотителя, как это следует из соотношения (1), должна быть равна $f_{a 1 m}=\left(p_{v}^{2}+Y_{g 2}^{2}\right)^{1 / 2}$ и значительно превышать величину $p_{v}$. При этом коэффициент поглощения $\mathrm{THz}$ излучения будет задан соотношением $A=2 /\left[1+\left(1+Y_{g 2}^{2} / p_{v}^{2}\right)^{1 / 2}\right]$. В частности, для $\lambda=100 \mu \mathrm{m}, d_{g}=2.5 \mu \mathrm{m}, \theta_{v}=0$ получим: $p_{v}=Y_{v}, \quad Y_{g 2} / Y_{v} \approx 6.3, \quad f_{a 1 m} / Y_{v} \approx 6.4, \quad R_{a}=1 / f_{a 1 m} \approx$ $\approx 59 \Omega / \square, A \approx 0.27$, что в три раза превышает значение $A$, соответствующее поглотителю со слоевой проводимостью $f_{a 1}=Y_{v}$.

В ИК диапазоне на некоторых длинах волн $\lambda \leq 4 d_{g}$ адмиттанс $Y_{g 2}$ может стать равным нулю и выполнится первое условие (2), однако второе условие будет нарушено, поскольку для бездисперсионного поглотителя $f_{a 1 m} / Y_{v}$ остается значительно больше единицы во всем диапазоне частот. При этом коэффициент поглощения не может превысить значения $A=4 p_{v} f_{a 1 m} /\left(p_{v}+f_{a 1 m}\right)^{2}$, которое для рассмотренного здесь примера равно 0.47 . Ниже мы покажем, что при наличии соответствующей частотной дисперсии слоевой проводимости поглотителя можно достичь полного поглощения в ИК диапазоне при повышенном коэффициенте поглощения в $\mathrm{THz}$ диапазоне.

\section{Примеры поглотителей излучения с частотной дисперсией проводимости}

Частотной дисперсией проводимости в ИК и $\mathrm{THz}$ областях обладают полупроводники, металлы, нанотрубки, графен и другие материалы [11]. Анализ влияния частотной дисперсии проводимости поглотителя на спектральную зависимость коэффициента поглощения в болометрических структурах проведем на примере трех типов материалов. Первый - высокоомные металлы и их сплавы, такие как Ti, TiAlV и $\mathrm{NiCr}$, обладающие слабой частотной дисперсией проводимости в ИК и $\mathrm{THz}$ диапазоне и традиционно используемые для изготовления поглотителей ИК и $\mathrm{THz}$ излучения. Второй низкоомные металлы, такие как $\mathrm{Al}, \mathrm{W}, \mathrm{Mo}, \mathrm{Cu}, \mathrm{Au}$, $\mathrm{Ni}$, проводимость которых существенно изменяется при переходе от терагерцового диапазона к инфракрасному и ее частотная зависимость удовлетворительно описывается моделью Друде со временем релаксации электронов порядка или более $10^{-14} \mathrm{~s}[12-14]$. Третий - слои одностенных углеродных нанотрубок (ОУНТ), в которых 
Таблица 1. Параметры модели Друде-Лоренца для слоев ОУНТ

\begin{tabular}{c|c|c|c}
\hline$j$ & $\omega_{p j}, \mathrm{~cm}^{-1}$ & $\omega_{j}, \mathrm{~cm}^{-1}$ & $\gamma_{j}, \mathrm{~cm}^{-1}$ \\
\hline 0 & 1926 & 0 & 2232 \\
1 & 3226 & 66 & 187 \\
2 & 4600 & 5910 & 1310 \\
3 & 4076 & 10435 & 2571 \\
4 & 1725 & 14772 & 1355
\end{tabular}

трубки самоорганизованы в виде пучков, содержащих от 100 до 500 трубок $[15,16]$.

Удельная проводимость многих металлов значительно изменяется при уменьшении толщины слоя ниже нескольких десятков нанометров [17]. В работе [18] показано, что проводимость слоя золота при толщинах менее $20 \mathrm{~nm}$ описывается соотношением

$$
\sigma(\omega)=\frac{\varepsilon_{0} \omega_{p}^{2} \tau}{1-i \omega \tau}\left[1+\frac{c}{1-i \omega \tau}\right]
$$

где $\omega_{p}-$ плазменная частота, $\omega-$ угловая частота; $\tau$ - время релаксации электронов проводимости. Значения $\omega_{p}$ и параметра $c$ зависят от толщины слоя золота и даны в указанной выше работе. Для толщины слоя $8 \mathrm{~nm}$, выбранного для моделирования поглотителя с частотной дисперсией проводимости, $\omega_{p} / 2 \pi \approx 630 \mathrm{THz}$, $\tau=18 \cdot 10^{-15}$ s и $c=-0.15$. При этом низкочастотное слоевое сопротивление слоя золота составляет $59 \Omega / \square$. В дальнейшем данный слой будем обозначать как AU.

Диэлектрическая проницаемость вышеуказанных слоев ОУНТ, полученных методом лазерной абляции, описывается моделью Друде-Лоренца [15]

$$
\varepsilon(\omega)=\varepsilon_{h}-\sum_{j=0}^{4} \frac{\omega_{p j}^{2}}{\omega^{2}-\omega_{j}^{2}+i \omega \gamma_{j}},
$$

где $\varepsilon_{h}-$ высокочастотное значение диэлектрической проницаемости, $\omega_{p j}-$ сила осциллятора, $\omega_{j}-$ центральная частота, $\gamma_{j}-$ спектральная ширина. При этом удельная проводимость ОУНТ задана соотношением $\sigma(\omega)=-i \varepsilon_{0} \omega\left[\varepsilon(\omega)-\varepsilon_{h}\right]$. В расчетах использовались данные, представленные в работе [15] для образца „В“ при температуре $300 \mathrm{~K}$ (табл. 1), $\varepsilon_{h}=1.04$. Здесь, так же как и в случае слоя золота, используются обозначения величин и их размерности, принятые в первоисточнике. В дальнейшем слои ОУНТ толщиной 0.12 и $0.25 \mu \mathrm{m}$ будем обозначать как NT12 и NT25 соответственно.

В поглотителе излучения кроме указанных выше слоев AU, NT12 и NT25 будут применяться также два слоя металла, не обладающие частотной дисперсией проводимости и характеризующиеся слоевым сопротивлением $R_{a}$, равным 50 или $377 \Omega / \square$. Для них будем использовать обозначение ND50 и ND377.

\section{Результаты и их обсуждение}

Расчет спектральных зависимостей коэффициента поглощения проводился для болометрической структуры (рис. 1), состоящей из термочувствительной мембраны, включающей в себя поглотитель излучения 1 и термочувствительный элемент 2 из оксида ванадия $\mathrm{VO}_{x}$ толщиной $100 \mathrm{~nm}$, расположенный между двумя диэлектрическими слоями оксинитрида кремния $3, \mathrm{SiN}_{x} \mathrm{O}_{y}$, каждый из которых имеет толщину $100 \mathrm{~nm}$. Мембрана с помощью проводящих теплоизолирующих балок подвешена над отражателем (зеркалом) 4, нанесенным на подложку 5, образуя вакуумный зазор 6 толщиной $3.5 \mu \mathrm{m}$, который вместе со слоями 2 и 3 выполняет роль оптического резонатора. Слоевое сопротивление отражателя равно $0.2 \Omega / \square$ при его толщине $200 \mathrm{~nm}$, показатель преломления оксида ванадия $\mathrm{VO}_{x}$ принимался равным 2.5 .

Будем рассматривать четыре типа поглотителя. Три из них содержат один из перечисленных выше проводящих слоев ND50, AU, NT25, пассивированных снаружи слоем оксинитрида кремния 7 толщиной $50 \mathrm{~nm}$ (рис. 1,a). Четвертый поглотитель (рис. $1, b)$ кроме слоя NT12, также пассивированного снаружи слоем $\mathrm{SiN}_{x} \mathrm{O}_{y}$, содержит дополнительный слой ND377, который отделен от слоя NT12 слоем оксинитрида кремния 7 толщиной $50 \mathrm{~nm}$ и позволяет эффективно управлять слоевой проводимостью поглотителя в ИК области, практически не изменяя eе в $\mathrm{THz}$ области. Двухслойный поглотитель будем обозначать как NT12/ND377.

Диэлектрическая проницаемость оксинитрида кремния описывалась моделью Друде-Лоренца (3) с параметрами, определенными по измеренным ИК спектрам отражения и пропускания слоя $\mathrm{SiN}_{x} \mathrm{O}_{y}$ толщиной $170 \mathrm{~nm}$, нанесенного на германиевую подложку, и приведенными

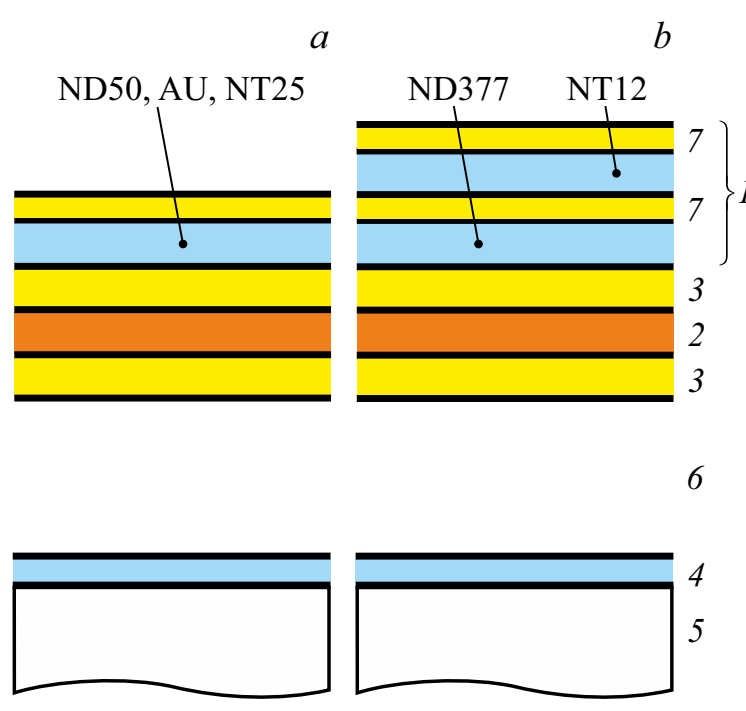

Рис. 1. Болометрический приемник ИК и ТНz излучения с однослойным $(a)$ и двухслойным $(b)$ поглотителем излучения (1), обладающим частотной дисперсией проводимости. Остальные пояснения обозначений - в тексте. 
Таблица 2. Параметры модели Друде-Лоренца для слоев $\mathrm{SiN}_{x} \mathrm{O}_{y}$

\begin{tabular}{c|c|c|c}
\hline$j$ & $\omega_{p j}, \mathrm{~cm}^{-1}$ & $\omega_{j}, \mathrm{~cm}^{-1}$ & $\gamma_{j}, \mathrm{~cm}^{-1}$ \\
\hline 1 & 7620 & 860 & 150 \\
2 & 7680 & 980 & 210
\end{tabular}

в табл. 2, $\varepsilon_{h}=2.6$. Для анализа влияния достаточно сильного поглощения ИК излучения слоями $\mathrm{SiN}_{x} \mathrm{O}_{y}$ в диапазоне длин волн 9-13 $\mu \mathrm{m}$ расчет также проводился при $\omega_{p j}=0$, что позволяло моделировать болометрический приемник, выполненный на основе аморфного кремния, в котором поглощение ИК излучения практически отсутствует.

На рис. 2 приведены спектральные зависимости активной $f_{a 1}$ и реактивной $f_{a 2}$ компонент слоевой проводимости поглотителя и взятой с обратным знаком реактивной компоненты адмиттанса оптического резонатора $Y_{g 2}$, нормированных на адмиттанс вакуума $Y_{v}$. Адмиттанс $Y_{g}$ рассчитывали, используя эффективную оптическую толщину резонатора, равную $4.1 \mu \mathrm{m} \mathrm{и}$ учитывающую толщины и высокочастотные показатели преломления всех слоев, расположенных между отражателем и поглотителем. Для всех рассмотренных на рис. 2 типов поглотителей, обладающих частотной дисперсией проводимости, первое из условий (2) выполняется вблизи длин волн 5 и $10 \mu \mathrm{m}$ (точки пересечения спектральных зависимостей $-Y_{g 2}$ и $\left.f_{a 2}\right)$. При этом на длинах волн, близких к $10 \mu \mathrm{m}$, величина $f_{a 1} / Y_{v}$ (и соответственно $f_{a 1} / p_{v}$ ) близка к единице и коэффициент поглощения $A \approx 100 \%$ (рис. 3-5). Для поглотителя $\mathrm{ND} 50$, не обладающего частотной дисперсией проводимости, первое из условий (2) выполняется на длинах волн 5.5 и $16.5 \mu \mathrm{m}$, а второе условие достаточно сильно нарушается и коэффициент поглощения $A$ составляет около 40\% (рис. 3). Поглотители AU и NT25 не содержат дополнительного бездисперсионного слоя и их активные компоненты на частоте, соответствующей $\lambda=5 \mu \mathrm{m}$, оказываются излишне занижены и составляют $0.18 Y_{v}$ и $0.25 Y_{v}$ соответственно, что приводит к понижению коэффициента поглощения до 0.5 и 0.7 на данной длине волны (рис. 3-5). Наличие дополнительного бездисперсионного слоя в поглотителе NT12/ND377 обеспечивает практически полное поглощение как на 5, так и на $10 \mu \mathrm{m}$, при этом пик поглощения вблизи $10 \mu \mathrm{m}$ становится более широким (рис. 4,5 ).

Отметим две характерные особенности, наблюдаемые на спектральных зависимостях коэффициента поглощения (рис. 3-5). Первая - практически полное отсутствие поглощения излучения на длинах волн, близких к $8 \mu \mathrm{m}$, - обусловлена совпадением положений поглотителя и узла электрического поля стоячей электромагнитной волны, образованной падающим на болометр и отраженным от зеркала излучением, и соответствует резкому возрастанию модуля реактивной компоненты адмиттанса оптического резонатора $\left|Y_{g 2}\right|$ (рис. 2). Вторая - слабое изменение коэффициента поглощения в достаточно большом интервале длин волн (от 20-30 до $100-200 \mu \mathrm{m})$ - обусловлена тем, что изменяющийся с длиной волны модуль реактивной компоненты адмиттанса оптического резонатора $\left|Y_{g 2}\right|$ в указанном диапазоне длин волн не превышает активной компоненты слоевой проводимости поглотителя $f_{a 1}$, как это происходит при значениях $f_{a 1}$, близких к единице, и потому не является определяющим в знаменателе соотношения (1), что позволяет при соответствующем выборе частотных зависимостей $f_{a 1}$ и $f_{a 2}$ достичь повышенного значения коэффициента поглощения в большом интервале длин волн и, следовательно, обеспечить его медленно изменение. Это особенно наглядно проявляется в случае бездисперсионного поглотителя ND50, в котором для диапазона длин волн от 5 до $100 \mu \mathrm{m}$ (за исключением длин волн, близких к $8 \mu \mathrm{m})$ определяющим параметром

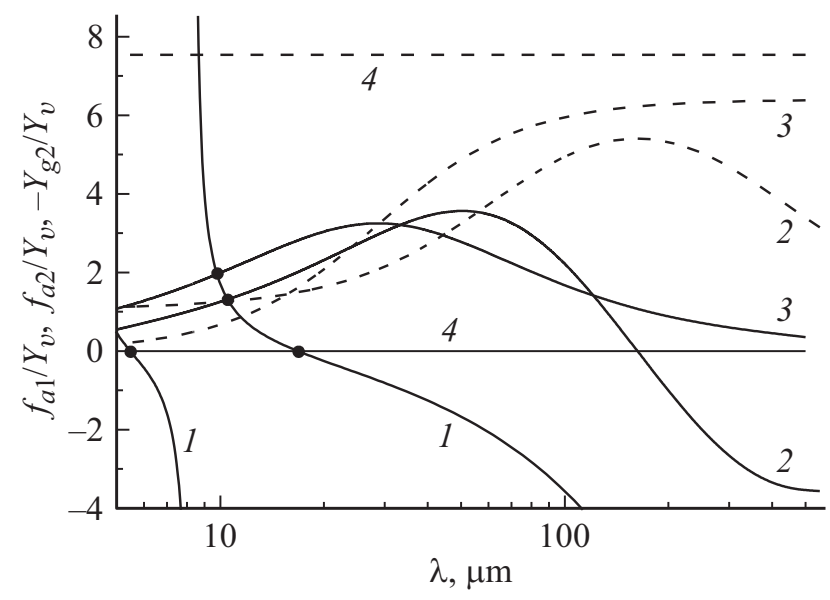

Рис. 2. Спектральные зависимости реактивной компоненты адмиттанса оптического резонатора $Y_{g 2}(1)$, активной $f_{a 1}$ (штрих) и реактивной $f_{a 2}$ (сплошная) компонент слоевой проводимости поглотителей NT12/ND377 (2), AU (3), ND50 (4), нормированных на адмиттанс вакуума $Y_{v}$.

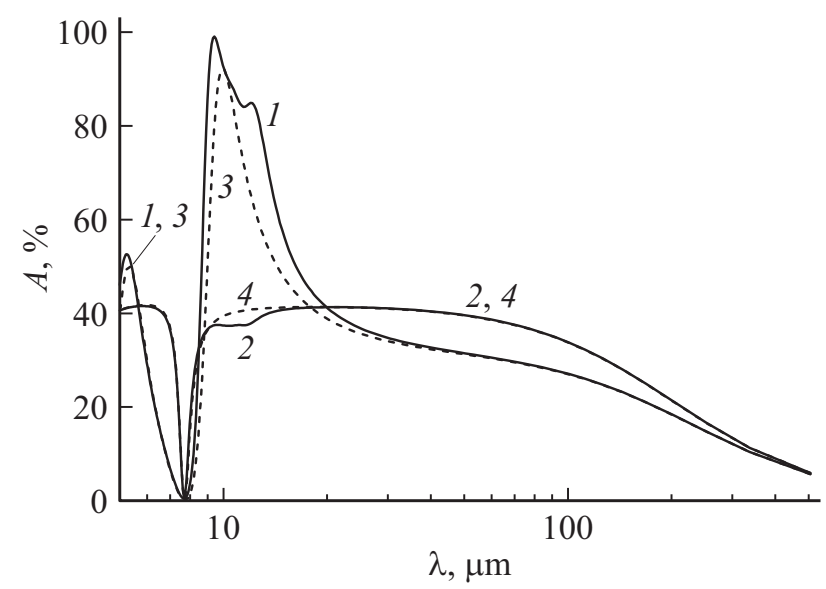

Рис. 3. Спектральные зависимости коэффициента поглощения в болометре с поглотителями $\mathrm{AU}(1,3)$ и $\mathrm{ND} 50(2,4)$ с учетом $(1,2)$ и без учета $(3,4)$ поглощения в слоях $\mathrm{SiN}_{x} \mathrm{O}_{y}$. 


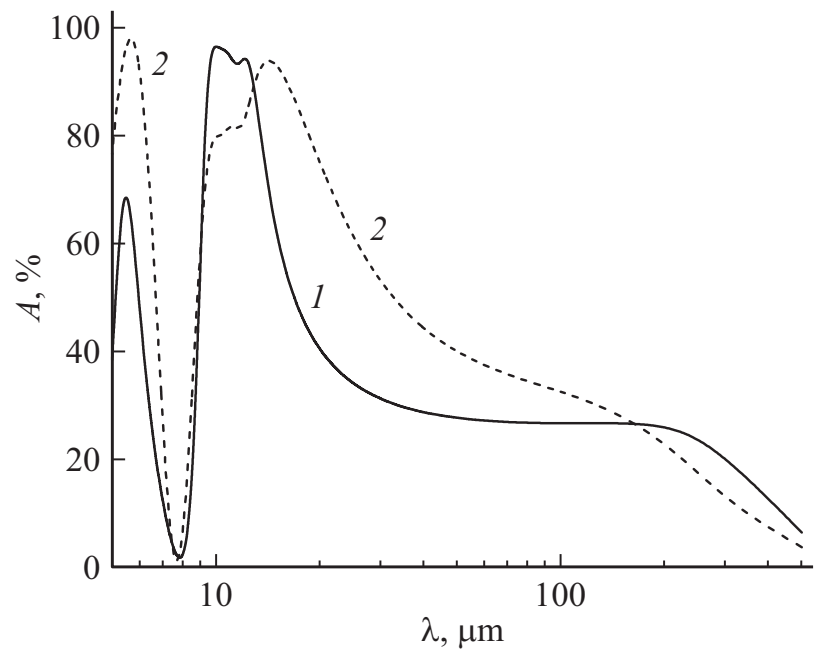

Pис. 4. Спектральные зависимости коэффициента поглощения в болометре с поглотителями NT25 (1), NT12/ND377 (2) при учете поглощения в слоях $\mathrm{SiN}_{x} \mathrm{O}_{y}$.

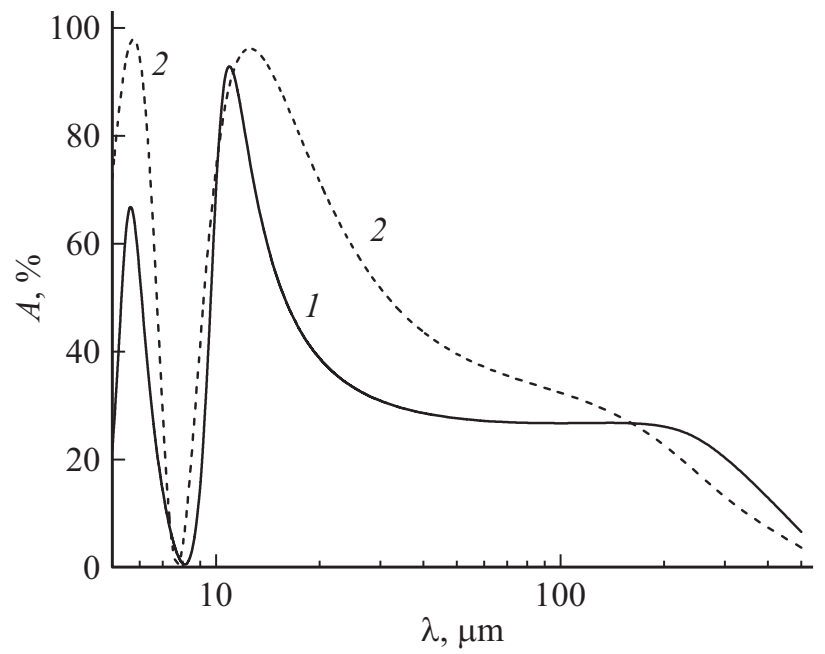

Рис. 5. Спектральные зависимости коэффициента поглощения в болометре с поглотителями NT25 (1), NT12/ND377 (2) без учета поглощения в слоях $\mathrm{SiN}_{x} \mathrm{O}_{y}$.

является частотно независимая активная компонента слоевой проводимости поглотителя $f_{a 1}$ (рис. 2), что и обеспечивает постоянство коэффициента поглощения в указанном интервале длин волн. При длинах волн более $100 \mu \mathrm{m}$ значение $\left|Y_{g 2}\right|$ превышает $f_{a 1}$, что приводит к уменьшению коэффициента поглощения.

Поглотитель излучения NT25 (рис. 4, 5), выполненный на основе слоя ОУНТ, обеспечивает заметно большее поглощение на длинах волн 200-300 $\mu \mathrm{m}$ по сравнению с поглотителями ND50 и AU (рис. 3). Это обусловлено тем, что поглотитель NT25 на этих длинах волн обладает большой отрицательной реактивной компонентой слоевой проводимости $f_{a 2}$, характерной для локализованных осцилляторов Лоренца, которая частично компенсирует положительную реактивную компоненту оптического резонатора $Y_{g 2}$ (рис. 2).

Выше было отмечено, что диэлектрические слои, выполненные из оксинитрида кремния, эффективно поглощают ИК излучения в диапазоне длин волн 9-13 $\mu \mathrm{m}$ и, следовательно, обладают высокочастотной проводимостью, которая приводит к значительному изменению как активной, так и реактивной составляющей эффективной слоевой проводимости поглотителя в указанном диапазоне частот. В результате чего при наличии дополнительного бездисперсионного слоя ND377 активная компонента эффективной слоевой проводимости поглотителя в указанном диапазоне длин волн может заметно превысить оптимальное значение, что приведет к понижению коэффициента поглощения. Из рис. 4 и 5 видно, что наличие поглощения в слоях $\operatorname{SiN}_{x} \mathrm{O}_{y}$ в случае применения поглотителя NT12/ND377 приводит к понижению коэффициента поглощения до $80 \%$ в области длин волн 10-12 $\mu \mathrm{m}$ (рис. 4), в то время как вне этой области (рис. 4) или в отсутствие поглощения в слоях $\mathrm{SiN}_{x} \mathrm{O}_{y}$ (рис. 5) коэффициент поглощения может составить 93 и 96\% соответственно.

\section{Заключение}

Проведен анализ влияния частотной дисперсии проводимости поглотителя на спектральную зависимость коэффициента поглощения болометрического приемника инфракрасного и терагерцового излучения. Показано, что в отличие от традиционно применяемых поглотителей на основе высокоомных металлов, не обладающих частотной дисперсией проводимости, применение поглотителей, выполненных из тонких слоев золота или одностенных углеродных нанотрубок, позволяет достичь практически полного поглощения излучения в ИК области (вблизи $10 \mu \mathrm{m})$ при сохранении повышенной чувствительности в $\mathrm{THz}$ области (вблизи $100 \mu \mathrm{m}$ ). Данный результат достигается без применения дополнительных резонаторов и при высоте подвески болометра, не превышающей 4-5 $\mu \mathrm{m}$, что позволяет обеспечить высокий процент выхода годных приемников. Показано, что применение поглотителей, состоящих из двух слоев, проводимости которых обладают различными частотными зависимостями, позволяет эффективно управлять спектральными зависимостями суммарной проводимости поглотителя и коэффициента поглощения болометрического приемника.

\section{Список литературы}

[1] Lee A.W.M., Hu Q. // Opt. Lett. 2005. Vol. 30. N 19. P. 2563-2565. DOI: $10.1364 /$ OL.30.002563

[2] Lee A.W.M., Williams B.S., Kumar S., Hu Q., Reno J.L. // IEEE Photonics Technology Letters. 2006. Vol. 18. N 13. P. 1415-1417. DOI: 10.1109/LPT.2006.877220

[3] Dem'yanenko M.A., Esaev D.G., Knyazev B.A., Kulipanov G.N., Vinokurov N.A. // Appl. Phys. Lett. 2008. Vol. 92. N 13. 131116. DOI: $10.1063 / 1.2898138$ 
[4] Oda N., Yoneyama H., Sasaki T., Sano M., Kurashina S., Hosako I., Sekine N., Sudoh T., Irie T. // Proc. SPIE. 2008. Vol. 6940. P. 69402Y. DOI: $10.1117 / 12.781630$

[5] Oda N. // C. R. Physique. 2010. Vol. 11. N 7-8. P. 496-509. DOI: $10.1016 /$ j.crhy.2010.05.001

[6] Nemoto N., Kanda N., Imai R., Konishi K., Miyoshi M., Kurashina S., Sasaki T., Oda N., Kuwata-Gonokami M. // IEEE Trans. on Terahertz Sci. Technol. 2016. Vol. 6. N 2. P. 175-182. DOI: 10.1109/TTHZ.2015.2508010

[7] Oda N., Sano M., Sonoda K., Yoneyama H., Kurashina S., Miyoshi M., Sasaki T., Hosako I., Sekine N., Sudou T., Ohkubo S. // Proc. SPIE. 2011. Vol. 8012. P. 80121B. DOI: $10.1117 / 12.888992$

[8] Демьяненко М.А. // ЖТФ. 2018. Т. 88. Вып. 1. С. 121-126. DOI: 10.21883/JTF.2018.01.45495.2165 [Dem'yanenko M.A. Techn. Phys. 2018. Vol. 63. N 1. P. 120-125. DOI: $10.1134 / \mathrm{S} 1063784218010097]$

[9] Борн М., Воль $\varnothing$ Э. Основы оптики. М.: Наука, 1973. 720 с. [Born M., Wolf E. Principles of Optics. Pergamon Press, Oxford, 1968.]

[10] Демьяненко М.А. // Оптич. журн. 2017. Т. 84. Вып. 1. C. 48-57. [Dem'yanenko M.A. J. Optical Technol. 2017. Vol. 84. N 1. P. 34-40. DOI: 10.1364/JOT.84.000034]

[11] Lloyd-Hughes J., Jeon T.I. // J. Infrared, Millimetre Terahertz Waves. 2012. Vol. 33. N 9. P. 871-925. DOI: $10.1007 / \mathrm{s} 10762-012-9905-\mathrm{y}$

[12] Ordal M.A., Bell R.J., Alexander R.W.Jr., Newquist L.A., Querry M.R. // Appl. Opt. 1988. Vol. 27. N 6. P. 1203-1209. DOI: $10.1364 / A O .27 .001203$

[13] Ordal M.A., Long L.L., Bell R.J., Bell S.E., Bell R.R., Alexander R.W.Jr., Ward C.A. // Appl. Opt. 1983. Vol. 22. N 7. P. $1099-1119$. DOI: $10.1364 / A O .22 .001099$

[14] Gall D. // J. Appl. Phys. 2016. Vol. 119. N 8. 085101. DOI: $10.1063 / 1.4942216$

[15] Borondics F., Kamarás K., Nikolou M., Tanner D.B., Chen Z.H., Rinzler A.G. // Phys. Rev. B. 2006. Vol. 74. N 4. 045431. DOI: $10.1103 /$ PhysRevB.74.045431

[16] Thess A., Lee R., Nikolaev P., Dai H., Petit P., Robert J., Xu C., Lee Y.H., Kim S.G., Rinzler A.G., Colbert D.T., Scuseria G.E., Tomanek D., Fischer J.E., Smalley R.E. // Science. 1996. Vol. 273. N 5274. P. 483-487. DOI: $10.1126 /$ science. 273.5274 .483

[17] Camacho M., Oliva A.I. // Microelectronics Journ. 2005. Vol. 36. N 3-6. P. 555-558. DOI: 10.1016/j.mejo.2005.02.068

[18] Walther M., Cooke D.G., Sherstan C., Hajar M., Freeman M.R., Hegmann F.A. // Phys. Rev. B. 2007. Vol. 76. N 12. P. 125408. DOI: $10.1103 /$ PhysRevB.76.125408 